\title{
Gene Expression Analysis in Response to Vernalization in Chinese Cabbage (Brassica rapa L.)
}

\author{
Ayasha Akter ${ }^{1,2 *}$, Junji Miyazaki ${ }^{3}$, Daniel J. Shea ${ }^{4}$, Namiko Nishida ${ }^{1}$, Satoko Takada ${ }^{1}$, \\ Naomi Miyaji', Hasan Mehraj', Motoki Shimizu', Md. Asad-ud Doullah', \\ Takeshi Takasaki-Yasuda $^{1}$, Keiichi Okazaki ${ }^{4}$ and Ryo Fujimoto ${ }^{1}$
}

\author{
${ }^{1}$ Graduate School of Agricultural Science, Kobe University, Kobe 657-8501, Japan \\ ${ }^{2}$ Department of Horticulture, Bangladesh Agricultural University, Mymensingh-2202, Bangladesh \\ ${ }^{3}$ Agriculture Victoria Research Division, Department of Jobs, Precincts and Regions, AgriBioscience, Bundoora, Victoria 3083, \\ Australia \\ ${ }^{4}$ Graduate School of Science and Technology, Niigata University, Niigata 950-2181, Japan \\ ${ }_{5}^{5}$ wate Biotechnology Research Center, Kitakami 024-0003, Japan \\ ${ }^{6}$ Department of Plant Pathology and Seed Science, Faculty of Agriculture, Sylhet Agricultural University, Sylhet-3100, Bangladesh
}

Chinese cabbage (Brassica rapa L. var. pekinensis) is an economically and agriculturally significant leafy vegetable that is extensively cultivated throughout the world. Vernalization is exposure to prolonged cold that alters gene expression and accelerates a transition from the vegetative to reproductive phase. Premature bolting caused by exposure to cold inhibits head production and reduces the yield of Chinese cabbage, and developing a late bolting line is important for breeding. Therefore, it is critical to identify the genes showing differential expression patterns during cold treatment in Chinese cabbage. However, there are few studies on the transcriptome profiling of different durations of cold treatments in Chinese cabbage. Here, we analyzed the gene expression profiles in a Chinese cabbage inbred line, RJKB-T24, given different durations of cold treatments using RNA sequencing. Differentially expressed genes (DEGs) between non-vernalized and vernalized samples tended to be downregulated, and some genes involved in the flowering pathway (including $B r F L C$ and $B r M A F$ genes) were downregulated following cold treatment. Functional enrichment analysis indicated that some DEGs were involved in the stress response and hormone signaling pathways. For genes involved in the FRI-containing complex, a known activator of FLC in Arabidopsis thaliana, only BrFRL1 showed changes in expression. In contrast to A. thaliana, $B r V I P$ and $B r V R N$ genes showed different expression patterns between paralogs during cold treatment, suggesting that Chinese cabbage's flowering pathway is somehow different and more complex than in A. thaliana. These outcomes provide significant insights into the genetic control of bolting and flowering that occur during the vernalization of Chinese cabbage.

Key Words: differentially expressed genes, flowering pathway, RNA-sequencing, transcriptome.

\section{Introduction}

Chinese cabbage (Brassica rapa L. var. pekinensis) is an important vegetable that contributes to human health

Received; October 2, 2019. Accepted; November 24, 2019.

First Published Online in J-STAGE on February 7, 2020.

This work was supported by a Grant-in-Aid for Scientific Research (B) $(15 \mathrm{H} 04433 \& 18 \mathrm{H} 02173)$ from the Japan Society for the Promotion of Science (JSPS), International Research Fellow of JSPS (Invitation Fellowships for Research in Japan (Long-term)), and Hyogo Science and Technology Association.

No conflicts of interest declared.

* Corresponding author (E-mail: aakterhort@bau.edu.bd). as a source of beneficial dietary fibers and vitamins. B. rapa is the first species in the genus Brassica to be sequenced, and a double haploid (DH) line of Chinese cabbage, chiifu-401-42, was examined by sequencing (Wang et al., 2011). Whole genome sequencing of this line showed that B. rapa experienced a whole genome triplication (WGT) after speciation between the genera Brassica and Arabidopsis (Cheng et al., 2012; Wang et al., 2011). Vernalization refers to prolonged exposure to cold temperature, and is usually required to facilitate flowering in Brassicaceae. Because Chinese cabbage can sense low temperatures during seed germination (seed-vernalization-responsive type), it sometimes un- 
dergoes early flowering/bolting due to low temperatures that occur during production in the fall (Shea et al., 2018; Su et al., 2018). Premature bolting leads to a decline in commercial quality and a loss of market value. When breeding Chinese cabbage cultivars, a high bolting resistance is preferred (Shea et al., 2018).

How vernalization promotes flowering in Arabidopsis thaliana has been extensively studied. Two genes, FRIGIDA (FRI) and FLOWERING LOCUS C $(F L C)$, play vital roles in the flowering process in A. thaliana. FRI encodes a novel protein with two coiled-coil domains and is an activator of $F L C$ (Johanson et al., 2000). FLC encodes a MADS-box transcription factor and acts as a suppressor of flowering (Michaels and Amasino, 1999; Sheldon et al., 1999). During cold treatment, FLC expression is decreased and maintained at the same level even after a return to warm conditions. VERNALIZATION INSENSITIVE 3 (VIN3), VERNALIZATION1 (VRN1), VRN2, and $V R N 5$ have been identified as regulatory genes of FLC expression associated with vernalization (Greb et al., 2007; He and Amasino, 2005; Wood et al., 2006). At the beginning of cold treatment, VIN3 is activated (Sung and Amasino, 2004), while expression of VRN1 and VRN2 does not change during cold treatment (Gendall et al., 2001; Levy et al., 2002). Five MADSbox genes, MADS AFFECTING FLOWERING1 (MAFI) to MAF5, belong to the MADS-box protein family containing FLC (Alvarez-Buylla et al., 2000; Ratcliffe et al., 2001, 2003) and act as floral repressors (Kim and Sung, 2010; Ratcliffe et al., 2001; Scortecci et al., 2001, 2003; Sheldon et al., 2009). MAF genes show temperature-dependent changes in expression; MAF1-MAF4 are downregulated by vernalization, whereas MAF5 is upregulated (Ratcliffe et al., 2001, 2003). MAFs also directly interact with each other and produce complexes that regulate the genes related to floral transition such as FLOWERING LOCUS T (FT) (Gu et al., 2013).

Transcriptional repression of $F L C$ during prolonged cold exposure is associated with changes in histone modifications from active marks (trimethylation of lysine 4 in histone $\mathrm{H} 3$ (H3K4me3) or H3K36me3) to a repressive mark (H3K27me3), initiating a conformational change from euchromatin to heterochromatin (Dennis and Peacock, 2007; Groszmann et al., 2011). This is mediated by Polycomb repressive complex 2 (PRC2) components including the $\mathrm{Su}(\mathrm{z}) 12$ homologue, VRN2, and two plant homeodomain (PHD) finger proteins, VRN5 and VIN3 (Bastow et al., 2004; Birve et al., 2001; De Lucia et al., 2008; Gendall et al., 2001; Sung and Amasino, 2004; Wood et al., 2006). Upon being returned to warm conditions after prolonged cold exposure, the PHD-PRC2 spreads across the FLC locus with spreading H3K27me3 marks, and the expression of FLC is epigenetically silenced (Angel et al., 2011; Finnegan and Dennis, 2007). VRN1 encodes a DNA- binding protein containing two plant-specific B3 domains, and is required for the increase in $\mathrm{H} 3 \mathrm{~K} 27 \mathrm{me} 3$ at the FLC locus during prolonged cold treatment (Levy et al., 2002; Sheldon et al., 2006).

In Chinese cabbage, the reduced transcription of FLC could be an important factor in premature bolting (Itabashi et al., 2018; Shea et al., 2018; Su et al., 2018; Takada et al., 2019). There are four FLC paralogs in B. rapa and at least three of them act as floral repressors (Kawanabe et al., 2016; Kim et al., 2007; Schranz et al., 2002; Takada et al., 2019). All FLC paralogs were downregulated by prolonged cold treatment, and an increased level of H3K27me3 was observed around the first exon in all FLC paralogs in Chinese cabbage (Akter et al., 2019; Kawanabe et al., 2016). Following a return to normal temperature growth conditions, H3K27me3 spreads along all four BrFLC paralogs providing stable repression of the gene (Akter et al., 2019; Takada et al., 2019). Many QTL analyses using populations derived from the crossing of parents with two different flowering times showed co-localization of QTLs and $F L C$ genes, suggesting that the different function of $F L C$ alleles between lines results in variations in flowering time (Kakizaki et al., 2011; Kitamoto et al., 2014; Kole et al., 2001; Li et al., 2009; Lou et al., 2007; Osborn et al., 1997; Schranz et al., 2002; Xiao et al., 2013; Zhao et al., 2010).

Innovations in sequencing technology have enabled us to examine the transcriptome by RNA-sequencing (RNA-seq), providing high accuracy, a wider detection range of transcription, and high reproducibility (Ansorge, 2009; Mortazavi et al., 2008; Saeki et al., 2016). Using this technology, transcriptional changes in response to many kinds of abiotic or biotic stresses have been examined in the genus Brassica (Long et al., 2015; Miyaji et al., 2017; Wang et al., 2016; Xing et al., 2016; Yong et al., 2014; Zou et al., 2013). This method has also been applied to the detection of transcriptional changes in Brassicaceae following vernalization in pakchoi (B. rapa var. chinensis) and radish (Raphanus sativus) (Liu et al., 2017; Sun et al., 2015).

There is no report examining the transcriptome associated with different durations of vernalization treatments in Chinese cabbage. In this study, we analyzed the transcriptome during vernalization by RNA-seq to discover genes that respond to cold treatment and to identify the metabolic pathways involved in the cold response in Chinese cabbage. Our study provides an important opportunity to facilitate the research on vernalization mechanisms in B. rapa.

\section{Materials and Methods}

\section{Plant materials and growth conditions}

A Chinese cabbage inbred line, RJKB-T24, was used as the plant material (Kawamura et al., 2016). Seeds were surface-sterilized and grown on agar solidified Murashige and Skoog (MS) medium with $1 \%(\mathrm{w} / \mathrm{v})$ 
sucrose under long day (LD) conditions (16 h light) at $22^{\circ} \mathrm{C}$. For cold treatment, 14-day seedlings on MS plates were treated for two $(2 \mathrm{~V})$, four $(4 \mathrm{~V})$, or six $(6 \mathrm{~V})$ weeks at $4{ }^{\circ} \mathrm{C}$ under the $\mathrm{LD}$ conditions (16 h light), or six weeks at $4^{\circ} \mathrm{C}$ and then seven days in normal growth conditions $(6 \mathrm{~V} 7 \mathrm{~N})$. RJKB-T24 did not flower without four weeks of cold treatment even if more than 100 days passed after sowing. When four weeks of cold treatment was applied to 14-day seedlings, flowering occurred 66 days after cold treatment. These results indicate that RJKB-T24 requires vernalization for flowering.

\section{$R N A$ extraction and $R T-P C R / q P C R$}

First and second leaves with $(2 \mathrm{~V}, 4 \mathrm{~V}, 6 \mathrm{~V}, 6 \mathrm{~V} 7 \mathrm{~N})$ or without (NV) cold treatments were used for isolation of total RNAs using the SV Total RNA Isolation System (Promega, Madison, WI, USA). The leaves from three individual plants in each condition were harvested as biological replicates. The cDNA was synthesized from $500 \mathrm{ng}$ total RNAs using ReverTra Ace qPCR RT Master Mix with gDNA Remover (TOYOBO Co., Ltd., Osaka, JAPAN). The cDNA was PCR amplified using Quick Taq ${ }^{\circledR}$ HS DyeMix (TOYOBO) for RT-PCR. Prior to quantitative real-time RT-PCR (RT-qPCR), the specificity of the primer set for each gene was first tested by electrophoresis of PCR-amplified products using QuickTaq ${ }^{\mathbb{R}}$ HS DyeMix (TOYOBO) on a $2.0 \%$ agarose gel in which single products were observed. Absence of genomic DNA contamination was confirmed by PCR of a no RT control using the BrACTIN (BrACT) gene (Fujimoto et al., 2006). PCR was performed using the following conditions; 1 cycle of $94^{\circ} \mathrm{C}$ for $2 \mathrm{~min}$ followed by 30 cycles of $94^{\circ} \mathrm{C}$ for $30 \mathrm{~s}, 55^{\circ} \mathrm{C}$ for $30 \mathrm{~s}$, and $68^{\circ} \mathrm{C}$ for $30 \mathrm{~s}$. Primer sequences used for RT-PCR are shown in Table S1.

RT-qPCR was performed by using LightCycler 96 (Roche Diagnostics International Ltd., Switzerland) and cDNA was amplified using FastStart Essential DNA Green Master (Roche). PCR conditions were $95^{\circ} \mathrm{C}$ for $10 \mathrm{~min}$ followed by 55 cycles of $95^{\circ} \mathrm{C}$ for $10 \mathrm{~s}, 60^{\circ} \mathrm{C}$ for $10 \mathrm{~s}$, and $72^{\circ} \mathrm{C}$ for $15 \mathrm{~s}$, and a melting program $\left(60^{\circ} \mathrm{C}\right.$ to $95^{\circ} \mathrm{C}$ at $0.1^{\circ} \mathrm{C} / \mathrm{s}$ ). After amplification cycles, each reaction was subjected to melt temperature analysis to confirm single amplified products. The expression level of each gene relative to $B r A C T$ was automatically calculated using automatic CQ calling according to the manufacturer's instructions (Roche). Data presented are the means and standard error (s.e.) calculated from three biological and experimental replications. Primer sequences used for RT-qPCR are shown in Table S1. Dunnett's test was performed for statistical analysis.

\section{RNA-sequencing}

RNA-sequencing (RNA-seq) was performed using first and second leaves with $(2 \mathrm{~V}, 4 \mathrm{~V}, 6 \mathrm{~V}, 6 \mathrm{~V} 7 \mathrm{~N})$ or without $(\mathrm{NV})$ cold treatments. A sequence library was prepared as described previously (Nagano et al., 2015). RNA-seq was performed with three replicates of each condition using Illumina Hiseq2000 (single-end sequencing, $50 \mathrm{bp}$ ). Sequence data have been submitted to the DDBJ database (http://www.ddbj.nig.ac.jp) under accession number DRA009156.

The quality of the RNA-seq libraries was evaluated using the FastQC software (http://www.bioinformatics. babraham.ac.uk/projects/fastqc/). After QC, filtered reads were used for further analysis. Tophat2 (Kim et al., 2013) with the default parameters was used to align RNA-seq reads against the B. rapa reference genome downloaded from the Ensemble Genome database (ftp://ftp.ensemblgenomes.org/pub/plants/release32/fasta/brassica_rapa/dna/Brassica_rapa.IVFCAASv1. dna.toplevel.fa). The levels of gene expression were scored by fragments per kilo-base per million (FPKM) using cufflinks, and cuffdiff was used for identification of differentially expressed genes (DEGs) with and without cold treatments (Trapnell et al., 2012). Analysis for enrichment of gene functional ontology terms was completed using the gene ontology (GO) tool agriGO (Du et al., 2010) according to methods we previously described (Shimizu et al., 2014). Statistical tests for enrichment of functional terms used the hypergeometric test and false discovery rate (FDR) correction for multiple testing to a level of $5 \%$ FDR.

We validated the RNA-seq data by RT-qPCR using 25 genes, and 23 of the 25 genes showed similar expression patterns to RNA-seq data (FPKM) (Fig. S1; Table S2).

\section{Results}

Identification of differentially expressed genes between vernalized and non-vernalized samples

We performed the RNA-seq in leaves with and without cold treatments in an inbred line of Chinese cabbage, RJKB-T24 that requires vernalization for flowering. Samples with three different durations of cold treatments, two $(2 \mathrm{~V})$, four $(4 \mathrm{~V})$, or six $(6 \mathrm{~V})$ weeks, and after a return to warm conditions for seven days following six weeks of cold treatment $(6 \mathrm{~V} 7 \mathrm{~N})$ were used. Total reads and mapped reads on the reference genome are shown in Table S3. The differentially expressed genes (DEGs) between non-vernalized (NV) and vernalized samples $(2 \mathrm{~V}, 4 \mathrm{~V}, 6 \mathrm{~V}, 6 \mathrm{~V} 7 \mathrm{~N})$ were identified by Tophat/cuffdiff at $95 \%$ confidence. A total of 3,787 (2V vs. NV), 3,543 (4V vs. NV), 2,630 (6V vs. $\mathrm{NV})$, and 3,896 (6V7N vs. NV) DEGs were identified, including 1,169, 1,291, 980, and 946 upregulated, and $2,618,2,252,1,650$, and 2,950 downregulated, genes in $2 \mathrm{~V}, 4 \mathrm{~V}, 6 \mathrm{~V}$, or $6 \mathrm{~V} 7 \mathrm{~N}$, respectively (Table 1 ).

Comparing DEGs between the different cold treatment conditions, 345 upregulated and 1,222 downregulated genes overlapped between $2 \mathrm{~V} / \mathrm{NV}$ and $4 \mathrm{~V} / \mathrm{NV}$, and the number of overlapped upregulated genes decreased between $2 \mathrm{~V} / \mathrm{NV}$ and $6 \mathrm{~V} / \mathrm{NV}$ and between 
2V/NV and $6 \mathrm{~V} 7 \mathrm{~N} / \mathrm{NV}$ (Fig. 1). The number of overlapped downregulated genes decreased between $2 \mathrm{~V} / \mathrm{NV}$ and $6 \mathrm{~V} / \mathrm{NV}$, whereas it increased between $2 \mathrm{~V} / \mathrm{NV}$ and $6 \mathrm{~V} 7 \mathrm{~N} / \mathrm{NV}$ (Fig. 1). The numbers of overlapped upregulated and downregulated genes between $4 \mathrm{~V} / \mathrm{NV}$ and $6 \mathrm{~V} / \mathrm{NV}$ were 322 and 934, respectively, and 277 upregulated and 1,207 downregulated genes overlapped between $4 \mathrm{~V} / \mathrm{NV}$ and $6 \mathrm{~V} 7 \mathrm{~N} / \mathrm{NV}$ (Fig. 1). Between 6V/NV and $6 \mathrm{~V} 7 \mathrm{~N} / \mathrm{NV}, 211$ upregulated and 936 downregulated genes overlapped (Fig. 1). There were 128 upregulated and 48 downregulated genes in three conditions $(2 \mathrm{~V} / \mathrm{NV}, 4 \mathrm{~V} / \mathrm{NV}$, and $6 \mathrm{~V} / \mathrm{NV})$, and 646 upregulated and 430 downregulated genes were found in four conditions $(2 \mathrm{~V} / \mathrm{NV}, 4 \mathrm{~V} / \mathrm{NV}, 6 \mathrm{~V} / \mathrm{NV}$, and 6V7N/NV) (Table 1).

Transcriptional changes in flowering pathway genes following cold treatment

Genes involved in flowering pathways in B. rapa have been identified (Wang et al., 2017), and 89 genes mainly involved in vernalization and autonomous pathways were selected in this study (Table S4). In our RNA-seq data, 29 out of 89 genes showed differential expression in vernalized samples compared with nonvernalized samples for at least one cold treatment condition (Fig. 2; Table S4). More genes showed downregulation than upregulation (Fig. 2; Table S4).

Expression levels of BrVIN3 (Bra020445) and SUPPRESSOR OF OVEREXPRESSION OF CO 1 (BrSOC1; Bra004928 and Bra039324) rose with longer cold treatment durations (Fig. 2). From RT-qPCR results, the expression of BrVIN3s decreased on return to normal temperature, while the increased expression of $B r S O C 1$ s was maintained (Fig. 3A). Three BrFLC paralogs (BrFLC1/Bra009055, BrFLC2/Bra028599, $B r F L C 3 / \mathrm{Bra} 006051)$ and three of five $B r M A F$ genes (Bra024350, Bra024351, Bra031888) were downregulated by cold treatment (Fig. 2), and repression of the expression levels was maintained after return to normal

Table 1. Number of differentially expressed genes in vernalized and non-vernalized samples.

\begin{tabular}{lcc}
\hline \hline & $\begin{array}{c}\text { Upregulated in } \\
\text { vernalized } \\
\text { samples }\end{array}$ & $\begin{array}{c}\text { Downregulated in } \\
\text { vernalized } \\
\text { samples }\end{array}$ \\
\hline 1. $2 \mathrm{~V}$ vs. $\mathrm{NV}(2 \mathrm{~V} / \mathrm{NV})$ & 1,169 & 2,618 \\
2. $4 \mathrm{~V}$ vs. $\mathrm{NV}(4 \mathrm{~V} / \mathrm{NV})$ & 1,291 & 2,252 \\
3. $6 \mathrm{~V}$ vs. NV $(6 \mathrm{~V} / \mathrm{NV})$ & 980 & 1,650 \\
4. $6 \mathrm{~V} 7 \mathrm{~N}$ vs. NV $(6 \mathrm{~V} 7 \mathrm{~N} / \mathrm{NV})$ & 946 & 2,950 \\
Overlapped genes $(1,2,3)$ & 128 & 48 \\
Overlapped genes $(1,2,3,4)$ & 646 & 430 \\
\hline
\end{tabular}

$\mathrm{NV}$, non-vernalized samples.

$2 \mathrm{~V}$, samples with two weeks of cold treatment.

$4 \mathrm{~V}$, samples with four weeks of cold treatment.

$6 \mathrm{~V}$, samples with six weeks of cold treatment.

$6 \mathrm{~V} 7 \mathrm{~N}$, six weeks of cold treatment followed by seven days under normal growth conditions. temperature (Fig. 2). Downregulation of the total amount of four BrFLC genes and three BrMAF genes was also confirmed by RT-qPCR (Fig. 3B).

From the RNA-seq data, we identified that $B r U B C 1$ (Bra026833), BrSEF (Bra006766), BrFRL1 (Bra008624), and one BrVRN1 (Bra022376) were upregulated by cold treatment (Figs. 2 and $\mathrm{S} 1$ ). $B r L D$ (Bra018540), BrUBC2 (Bra026582), one VERNALIZATION INDEPENDENCE 4 (BrVIP4) paralog (Bra029332), BrVIP5 (Bra031459), BrVIP6/BrELF8 (Bra013162), and two of three BrVRN1 paralogs



Fig. 1. The total number of overlapped genes under different vernalization conditions. $\mathrm{NV}$, non-vernalized; $2 \mathrm{~V}$, two weeks of cold treatment; $4 \mathrm{~V}$, four weeks of cold treatment; $6 \mathrm{~V}$, six weeks of cold treatment; $6 \mathrm{~V} 7 \mathrm{~N}$, six weeks of cold treatment followed by seven days under normal growth conditions.

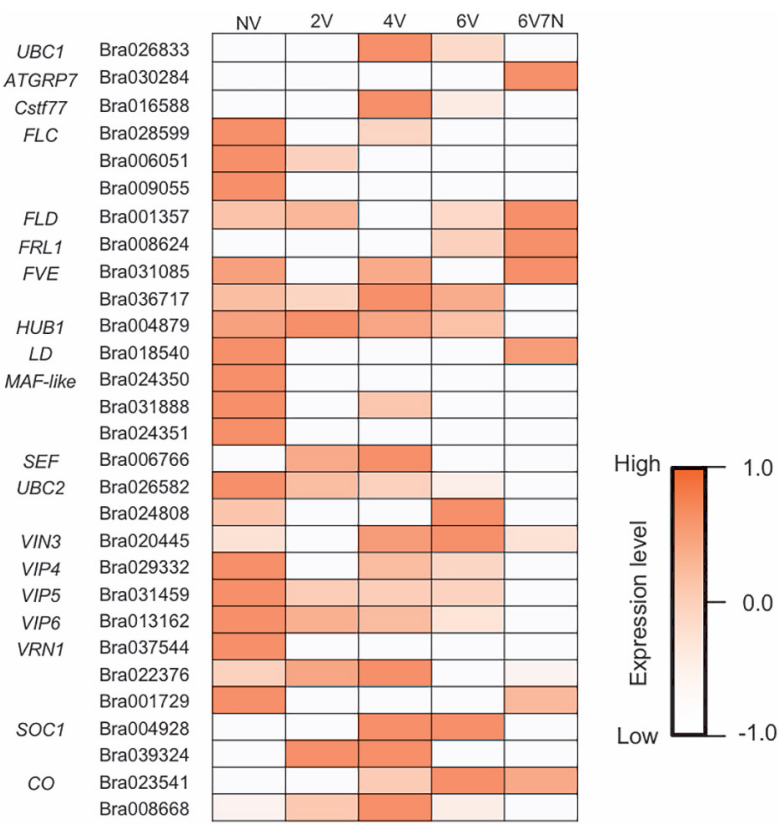

Fig. 2. Heat map of the expression levels in genes involved in the flowering pathway showing upregulation or downregulation by cold treatment. Expression levels (FPKM) higher or lower than the median are shown. NV, non-vernalized; $2 \mathrm{~V}$, two weeks of cold treatment; $4 \mathrm{~V}$, four weeks of cold treatment; $6 \mathrm{~V}$, six weeks of cold treatment; $6 \mathrm{~V} 7 \mathrm{~N}$, six weeks of cold treatment followed by seven days under normal growth conditions. 
A
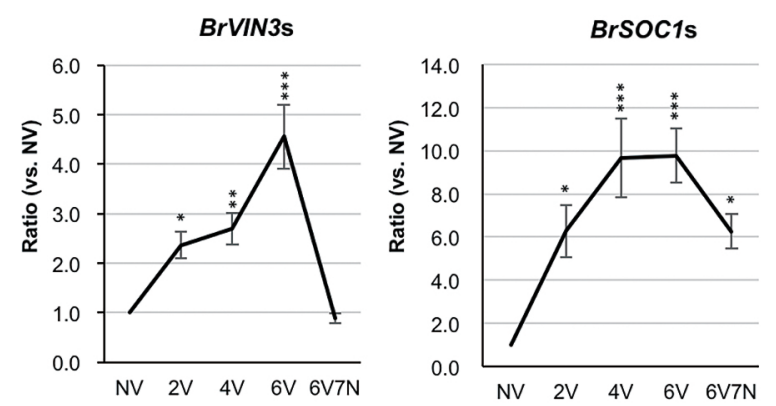

C

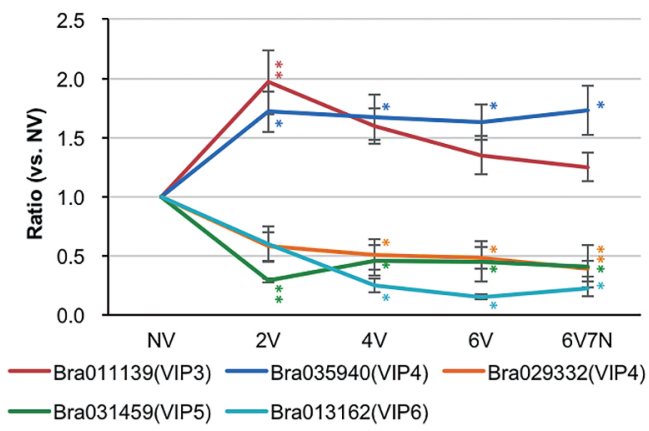

B

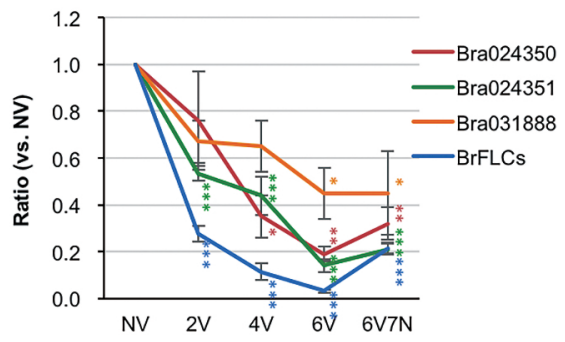

D

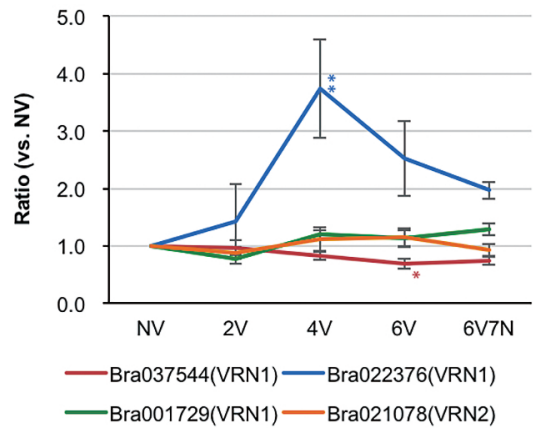

Fig. 3. Validation of the expression levels by RT-qPCR for selected genes involved in the flowering pathway. The expression levels of each gene relative to $\operatorname{BrACTIN}(B r A C T)$ were calculated, and the y-axis shows the ratio of vernalized samples $(2 \mathrm{~V}, 4 \mathrm{~V}, 6 \mathrm{~V}, 6 \mathrm{~V} 7 \mathrm{~N})$ compared with nonvernalized samples (NV). Data presented are the average and standard error (s.e.) from three biological and experimental replications. Statistical tests between non-vernalized and vernalized samples are shown (Dunnett's test, ${ }^{*} ; P<0.05, * * ; P<0.01, * * * ; P<0.001$ ). NV, nonvernalized; $2 \mathrm{~V}$, two weeks of cold treatment; $4 \mathrm{~V}$, four weeks of cold treatment; $6 \mathrm{~V}$, six weeks of cold treatment; $6 \mathrm{~V} 7 \mathrm{~N}$, six weeks of cold treatment followed by seven days under normal growth conditions.

(Bra037544, Bra001729) showed downregulation by cold treatment (Fig. 2). In addition, we found opposite expression patterns between BrVIP4 paralogs (Fig. 3C) and between BrVRN1 paralogs (Fig. 3D).

\section{Functional annotation of differentially expressed genes after vernalization}

To identify the functions of DEGs, GO enrichment analysis was conducted. The upregulated and downregulated genes at different durations of vernalization $(2 \mathrm{~V}$, $4 \mathrm{~V}, 6 \mathrm{~V}$, and $6 \mathrm{~V} 7 \mathrm{~N}$ ) were categorized into the $\mathrm{GO}$ cellular component (CC), GO molecular function (MF), and GO biological process (BP). In upregulated genes in $2 \mathrm{~V}, 4 \mathrm{~V}, 6 \mathrm{~V}$, and $6 \mathrm{~V} 7 \mathrm{~N}, 25,64,93$, and 22 categories were overrepresented $(P<0.0001$, FDR $<0.05)$, respectively (Tables S5-S8). Twelve categories were overrepresented in upregulated genes in $2 \mathrm{~V}, 4 \mathrm{~V}$, and $6 \mathrm{~V}$, and six categories were overrepresented in upregulated genes in all four conditions (Fig. S2). In downregulated genes in $2 \mathrm{~V}, 4 \mathrm{~V}, 6 \mathrm{~V}$, and $6 \mathrm{~V} 7 \mathrm{~N}, 344,372,241$, and 377 categories were overrepresented $(P<0.0001, \mathrm{FDR}<$ $0.05)$, respectively (Tables S5-S8). Ten categories were overrepresented in downregulated genes in $2 \mathrm{~V}, 4 \mathrm{~V}$, and $6 \mathrm{~V}$, and 73 categories were overrepresented in downregulated genes in all four conditions (Fig. S2).

The categories related to stress response such as
'Response to cold' or response to plant hormone stimulation such as 'Response to jasmonic acid stimulus', 'Response to ethylene stimulus', and 'Response to salicylic acid stimulus' tended to be overrepresented in both upregulated and downregulated genes by cold treatment (Figs. 4 and S2). The category 'Response to heat' tended to be overrepresented in upregulated genes, while the categories of 'Small molecule metabolic process' and 'Defense response' tended to be overrepresented in downregulated genes (Figs. 4 and S2). The category 'Regulation of transcription' was overrepresented in upregulated genes, especially at $4 \mathrm{~V}$ (Fig. 4).

We performed RT-qPCR to examine the expression of six genes related to stress response. The expression level of HEAT SHOCK TRANSCRIPTION FACTOR $B 2 A$ (BrHSFB2A) increased with cumulative cold treatment time, and its expression decreased when it returned to normal temperature (Fig. 5). The expression level of HEAT SHOCK PROTEIN 70 (BrHSP70) increased with cumulative cold treatment time, and its expression was maintained when it returned to normal temperature (Fig. 5). COLD REGULATED 15B $(B r C O R 15 B)$ showed rapid induction of its expression at low temperature and this expression was undetectable when it returned to normal temperature 


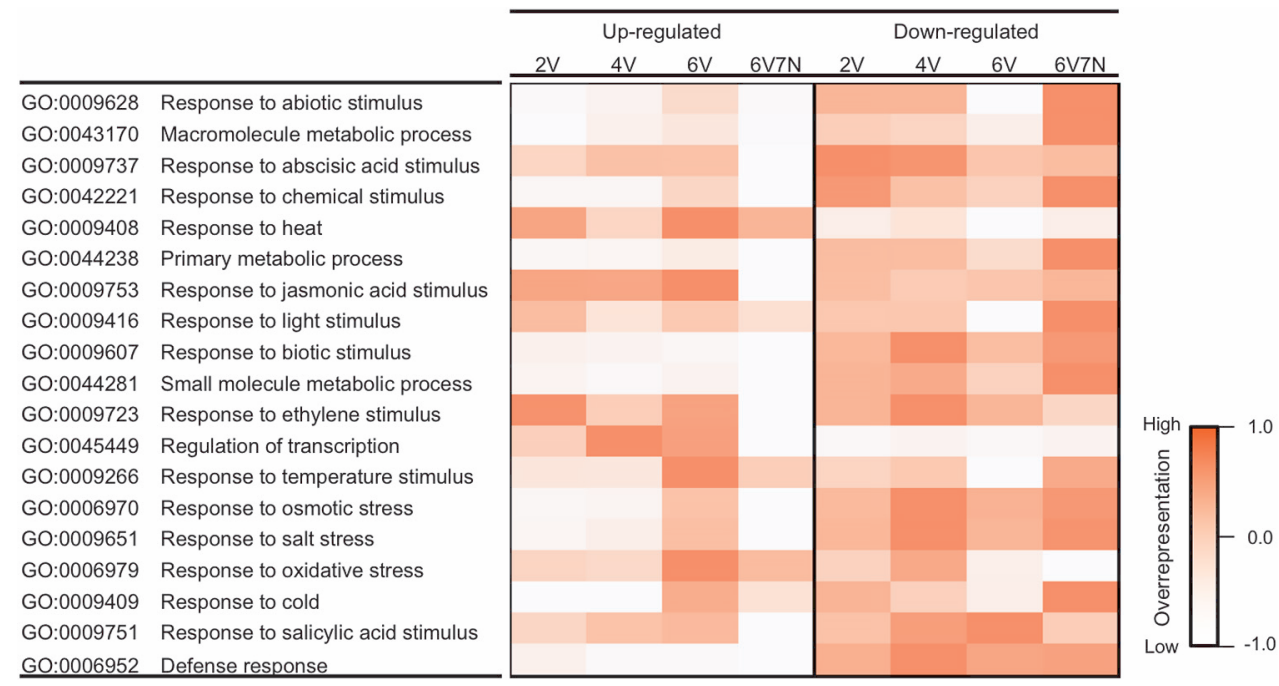

Fig. 4. GO enrichment analysis using upregulated or downregulated genes by cold treatment. The Log 10 score of the $p$-value shows overrepresentation. The heat map shows overrepresentation higher or lower than the median. All selected categories belong to the GO biological process (BP). NV, non-vernalized; $2 \mathrm{~V}$, two weeks of cold treatment; $4 \mathrm{~V}$, four weeks of cold treatment; $6 \mathrm{~V}$, six weeks of cold treatment; $6 \mathrm{~V} 7 \mathrm{~N}$, six weeks of cold treatment followed by seven days under normal growth conditions.

BrHSFB2A

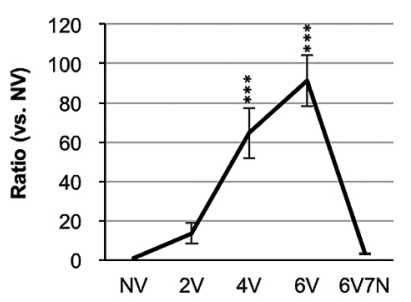

BrIAA28



BrHSP70

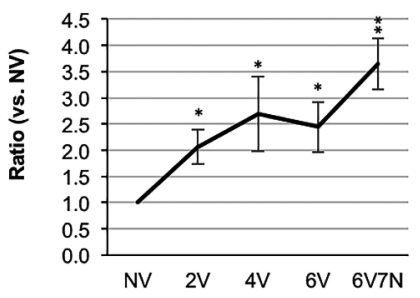

BrILL6

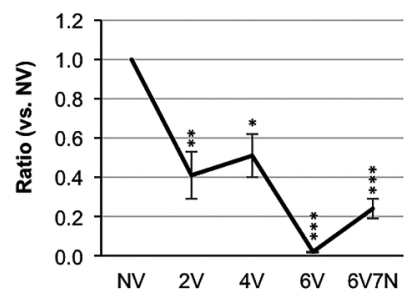

BrCOR15B

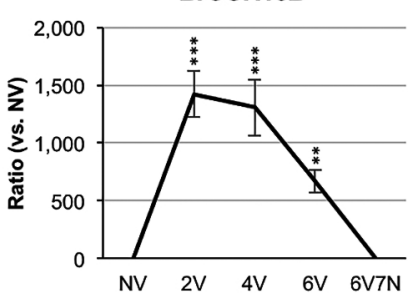

BrMYB28

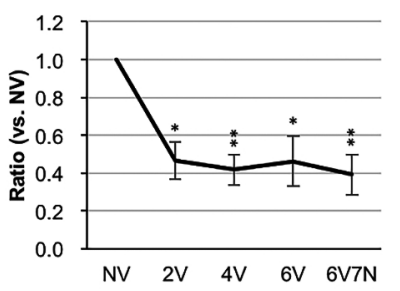

Fig. 5. The expression patterns of six genes related to stress response measured by RT-qPCR. The epression level of each gene relative to $\operatorname{BrACTIN}(B r A C T)$ was calculated. The relative expression levels of vernalized samples $(2 \mathrm{~V}, 4 \mathrm{~V}, 6 \mathrm{~V}, 6 \mathrm{~V} 7 \mathrm{~N})$ compared with non-vernalized samples (NV) are shown. Data presented are the average and standard error (s.e.) from three biological and experimental replications. Dunnett's test was performed to compare the expression levels between non-vernalized and vernalized samples. $* ; P<0.05, * * ; P<0.01$, ***; $P<0.001$. NV, non-vernalized; $2 \mathrm{~V}$, two weeks of cold treatment; $4 \mathrm{~V}$, four weeks of cold treatment; $6 \mathrm{~V}$, six weeks of cold treatment; $6 \mathrm{~V} 7 \mathrm{~N}$, six weeks of cold treatment followed by seven days under normal growth conditions.

(Fig. 5). Expression levels of INDOLE-3-ACETIC ACID INDUCIBLE 28 (BrIAA28), IAA-LEUCINE RESISTANT (ILR)-LIKE GENE 6 (BrILLO), and BrMYB28 were cumulatively downregulated by cold treatment, and repression of the expression was maintained after return to normal temperature (Fig. 5).

\section{Discussion}

Vernalization is one of the major pathways for flowering regulation, and there are variations in flowering times and vernalization requirements among different lines and cultivars of Chinese cabbage (Su et al., 2018). Previous research revealed that $B r F L C$ gene(s) is a key factor for vernalization in Chinese cabbage (Itabashi 
et al., 2018; Shea et al., 2018; Su et al., 2018; Takada et al., 2019). However, there is limited research showing how the regulators of $B r F L C$ genes react in response to vernalization or the transcriptional response at the whole genome level during prolonged cold treatment in Chinese cabbage.

In this study, we analyzed the transcriptome using first and second leaves before and after vernalization, and differentially expressed genes tended to be downregulated by cold treatment. During cold treatments, plant growth was slow, but leaf size gradually increased according to the low temperature period. In $6 \mathrm{~V} 7 \mathrm{~N}$, the plant growth rate returned to normal when the temperature was returned to that of normal growth conditions, and the leaf size of $6 \mathrm{~V} 7 \mathrm{~N}$ was larger than NV. Comparing the expression levels in vernalized samples $(2 \mathrm{~V}, 4 \mathrm{~V}$, $6 \mathrm{~V}, 6 \mathrm{~V} 7 \mathrm{~N})$ and non-vernalized samples $(\mathrm{NV})$, it was suggested that to some extent genes whose expression levels changed depending on the different growth stages were involved, especially in $6 \mathrm{~V} 7 \mathrm{~N}$. A cold responsive gene, BrCOR15B, was highly induced in response to cold treatment, but rapidly returned to previous expression levels upon return to normal growth conditions. $\mathrm{BrSOCl}$ genes were upregulated during cold treatment. RT-qPCR data showed that the $\mathrm{BrSOC1}$ expression level in $6 \mathrm{~V} 7 \mathrm{~N}$ was still higher than $\mathrm{NV}$, while RNA-seq data showed similar expression levels between $6 \mathrm{~V} 7 \mathrm{~N}$ and NV. The expression patterns of $\mathrm{BrSOCl}$ in previous reports in B. rapa were similar to the RT-qPCR results (Akter et al., 2019; Kawanabe et al., 2016), suggesting that the RT-qPCR results represent the correct expression pattern. The BrVIN3 gene was upregulated and $B r F L C$ genes were downregulated during cold treatment, consistent with previous studies in A.thaliana and B.rapa (Deng et al., 2011; Jung et al., 2018; Kawanabe et al., 2016; Sheldon et al., 2000, 2006). These results indicate that we could identify genes for which the expression level was changed by cold treatment.

GO categories related to stress or stimulus tended to be overrepresented in both upregulated and downregulated genes by cold treatment, but some GO categories showed a difference between upregulated and downregulated genes. The category 'Response to heat' was overrepresented in upregulated genes, and we showed that expression levels of BrHSFB2A and BrHSP70 increased during cold treatment. It has already been shown that $H_{S f s}$ and $H S P$ s, which were first described as factors that function under high temperature conditions, are induced at low temperatures (Swindell et al., 2007). These results suggest that upregulation of genes categorized as 'Response to heat' play a role in cold stress response. The category 'Regulation of transcription' was overrepresented in upregulated genes after four weeks of cold treatment, suggesting that some transcription factors are activated when a sufficient period of cold is applied. In contrast, the categories
'Response to biotic stimulus' and 'Defense response' were overrepresented in downregulated genes. Some studies have reported the cross-talk between biotic stress response and the transition to flowering (Chin et al., 2013; Lyons et al., 2015). The genes categorized as "response to plant hormone stimulation" tended to show altered the expression levels following cold treatment. We found the downregulation of genes was involved in auxin signaling, and previous studies showed that a decreased IAA hormone level was detected after vernalization in B. rapa (Huang et al., 2018a). Although we could not distinguish between genes involved in the vernalization response promoting flowering and the physical cold response, some genes identified in this study could be involved in the vernalization response or the transition from the vegetative to reproductive phase (e.g., $B \mathrm{SSOC1}$ ).

In A. thaliana, regulators of AtFLC have been identified (Kim and Sung, 2014). The FRI containing complex (FRI-C) including AtFRI, AtFRL1, AtFES1, AtSUF4, and AtFLX, and the PAF1 complex including AtVIP4, AtVIP5, AtELF7, and AtELF8 are known as activators of AtFLC expression (Kim and Sung, 2014; Shea et al., 2018). In this study, BrFRL1 expression levels were increased with cumulative cold treatment time, while other genes involved in FRI-C did not show any change in their expression levels during cold treatment. Expressions of BrFLC genes were downregulated with cumulative cold treatment time; therefore, the upregulation $B r F R L 1$ could be independent from the downregulation of $B r F L C$ genes during cold treatment. Genes involved in the PAF1 complex showed a change in expression levels during cold treatment in Chinese cabbage. BrVIP3 and one of two BrVIP4 genes showed upregulation by cold treatment, while one of two BrVIP4, BrVIP5, and BrVIP6 genes showed downregulation by cold treatment. In A. thaliana, vernalizationassociated changes in mRNA for AtVIP4, AtVIP5, or AtVIP6 have not been observed (Oh et al., 2004; Zhang and van Nocker, 2002). If the PAF1 complex is also an activator of BrFLC genes in Chinese cabbage, the downregulation of the genes involved in the PAF1 complex by vernalization could result in the downregulation of $B r F L C$ genes.

Genes involved in autonomous pathways or components of PHD-PRC2 are known as repressors of AtFLC expression in A.thaliana (Kim and Sung, 2014; Michaels and Amasino, 2001). Expression levels of AtVRN1 and AtVRN2 are not affected by vernalization in A. thaliana (Gendall et al., 2001; Levy et al., 2002). However, in this study, expression levels of BrVRN1 genes changed by cold treatment in Chinese cabbage, and the expression patterns observed during cold treatment were different among the three paralogous genes; two BrVRN1 genes were downregulated and one BrVRN1 gene was upregulated. Upregulation of BrVRN1 by 20 days of cold treatment was also ob- 
served in pak choi (Sun et al., 2015). These results suggest that, unlike in A.thaliana, it may be necessary to examine the possibility that the suppression of BrFLC expression is dependent on the upregulation of $B r V R N 1$ by cold in B. rapa.

Five $B r M A F$ genes were identified in the reference genome of $B$.rapa, but an orthologous relationship between BrMAF genes in B. rapa and AtMAF1-5 in A. thaliana was not able to be determined (Cheng et al., 2012). Two BcMAF genes in pak choi, BcMAF1 (homologous gene, Bra031884, blastp, $3 \mathrm{e}^{-61}$ ) and BcMAF2 (homologous gene, Bra031888, blastp, $2 \mathrm{e}^{-74}$ ), have been shown to act as floral repressors (Huang et al., 2018 b, 2019). In this study, we showed that three of five $B r M A F$ genes were downregulated with cumulative cold treatment time, suggesting that downregulation of $B r M A F$ genes is important for promotion of flowering by vernalization. Further research will be required to clarify how much the $B r M A F$ genes contribute to the promotion of flowering by vernalization in comparison with $B r F L C$ genes.

In this study, we identified differentially expressed genes following cold treatment and some genes involved in flowering pathways showed different expression patterns between Chinese cabbage and A. thaliana. In addition, in genes involved in the flowering pathways, we found a difference in expression patterns between paralogous genes during cold treatment in Chinese cabbage, resulting in a more complicated flowering pathway in Chinese cabbage than in A. thaliana. The findings in A. thaliana can mostly be applied to the molecular mechanism(s) of vernalization in Chinese cabbage, but there are some differences compared to A.thaliana; for example, cold-induced noncoding RNAs were not found in Chinese cabbage ( $\mathrm{Li}$ et al., 2016; Shea et al., 2019). Further analyses of the genes found in this study will be required to identify the genetic factors involved in vernalization and the precise molecular mechanism of vernalization in Chinese cabbage.

\section{Acknowledgements}

We thank Dr. Kenji Osabe for his helpful comments and manuscript editing.

\section{Literature Cited}

Akter, A., S. Takahashi, W. Deng, D. J. Shea, E. Itabashi, M. Shimizu, N. Miyaji, K. Osabe, N. Nishida, Y. Suzuki, C. A. Helliwell, M. Seki, W. J. Peacock, E. S. Dennis and R. Fujimoto. 2019. The histone modification H3 lysine 27 trimethylation has conserved gene regulatory roles in the triplicated genome of Brassica rapa L.. DNA Res. 26: 433-443.

Alvarez-Buylla, E. R., S. J. Liljegren, S. Pelaz, S. E. Gold, C. Burgeff, G. S. Ditta, F. Vergara-Silva and M. F. Yanofsky. 2000. MADS-box gene evolution beyond flowers: expression in pollen, endosperm, guard cell, roots and trichomes. Plant J. 24: 1-11.

Angel, A., J. Song, C. Dean and M. Howard. 2011. A polycomb- based switch underlying quantitative epigenetic memory. Nature 476: 105-108.

Ansorge, W. J. 2009. Next-generation DNA sequencing techniques. N. Biotechnol. 25: 195-203.

Bastow, R., J. S. Mylne, C. Lister, Z. Lippman, R. A. Martienssen and C. Dean. 2004. Vernalization requires epigenetic silencing of FLC by histone methylation. Nature 427: 164-167.

Birve, A., A. K. Sengupta, D. Beuchle, J. Larsson, J. A. Kennison, A. Rasmuson-Lestander and J. Müller. 2001. $\mathrm{Su}(z) 12$, a novel Drosophila Polycomb group gene that is conserved in vertebrates and plants. Development 128: 3371-3379.

Cheng, F., J. Wu, L. Fang, S. Sun, B. Liu, K. Lin, G. Bonnema and X. Wang. 2012. Biased gene fractionation and dominant gene expression among the subgenomes of Brassica rapa. PLoS One 7: e36442. DOI: 10.1371/journal.pone.0036442.

Chin, K., T. A. DeFalco, W. Moeder and K. Yoshioka. 2013. The Arabidopsis cyclic nucleotide-gated ion channels AtCNGC2 and AtCNGC4 work in the same signaling pathway to regulate pathogen defense and floral transition. Plant Physiol. 163: 611-624.

De Lucia, F., P. Crevillen, A. M. Jones, T. Greb and C. Dean. 2008. A PHD-polycomb repressive complex 2 triggers the epigenetic silencing of $F L C$ during vernalization. Proc. Natl. Acad. Sci. USA 105: 16831-16836.

Deng, W., H. Ying, C. A. Helliwell, J. M. Taylor, W. J. Peacock and E. S. Dennis. 2011. FLOWERING LOCUS C (FLC) regulates development pathways throughout the life cycle of Arabidopsis. Proc. Natl. Acad. Sci. USA 108: 6680-6685.

Dennis, E. S. and W. J. Peacock. 2007. Epigenetic regulation of flowering. Curr. Opin. Plant Biol. 10: 520-527.

Du, Z., X. Zhou, Y. Ling, Z. Zhang and Z. Su. 2010. agriGO: a GO analysis toolkit for the agricultural community. Nucleic Acids Res. 38: W64-W70.

Finnegan, E. J. and E. S. Dennis. 2007. Vernalization-induced trimethylation of histone $\mathrm{H} 3$ lysine 27 at $F L C$ is not maintained in mitotically quiescent cells. Curr. Biol. 17: 19781983.

Fujimoto, R., T. Sasaki and T. Nishio. 2006. Characterization of DNA methyltransferase genes in Brassica rapa. Genes Genet. Syst. 81: 235-242.

Gendall, A. R., Y. Y. Levy, A. Wilson and C. Dean. 2001. The VERNALIZATION 2 gene mediates the epigenetic regulation of vernalization in Arabidopsis. Cell 107: 525-535.

Greb, T., J. S. Mylne, P. Crevillen, N. Geraldo, H. An, A. R. Gendall and C. Dean. 2007. The PHD finger protein VRN5 functions in the epigenetic silencing of Arabidopsis FLC. Curr. Biol. 17: 73-78.

Groszmann, M., I. K. Greaves, N. Albert, R. Fujimoto, C. A. Helliwell, E. S. Dennis and W. J. Peacock. 2011. Epigenetics in plants-vernalisation and hybrid vigour. Biochim. Biophys. Acta 1809: 427-437.

Gu, X., C. Le, Y. Wang, Z. Li, D. Jiang, Y. Wang and Y. He. 2013. Arabidopsis FLC clade members form floweringrepressor complexes coordinating responses to endogenous and environmental cues. Nat. Commun. 4: 1947.

He, Y. and R. M. Amasino. 2005. Role of chromatin modification in flowering-time control. Trends Plant Sci. 10: 30-35.

Huang, F., T. Liu and X. Hou. 2018b. Isolation and functional characterization of a floral repressor, $B c M A F 1$, from Pakchoi (Brassica rapa ssp. chinensis). Front. Plant Sci. 9: 290.

Huang, F., T. Liu, J. Tang, W. Duan and X. Hou. 2019. BcMAF2 activates BCTEMI and represses flowering in Pak-choi (Brassica rapa ssp. chinensis). Plant Mol. Biol. 100: 19-32.

Huang, F., X. Wu, X. Hou, S. Shao and T. Liu. 2018a. Vernaliza- 
tion can regulate flowering time through microRNA mechanism in Brassica rapa. Physiol. Plant. 164: 204-215.

Itabashi, E., K. Osabe, R. Fujimoto and T. Kakizaki. 2018. Epigenetic regulation of agronomical traits in Brassicaceae. Plant Cell Rep. 37: 87-101.

Johanson, U., J. West, C. Lister, S. Michaels, R. Amasino and C. Dean. 2000. Molecular analysis of FRIGIDA, a major determinant of natural variation in Arabidopsis flowering time. Science 290: 344-347.

Jung, W. Y., A. Lee, J. S. Moon, Y. S. Kim and H. S. Cho. 2018. Genome-wide identification of flowering time genes associated with vernalization and the regulatory flowering networks in Chinese cabbage. Plant Biotechnol. Rep. 12: 347363.

Kakizaki, T., T. Kato, N. Fukino, M. Ishida, K. Hatakeyama and S. Matsumoto. 2011. Identification of quantitative trait loci controlling late bolting in Chinese cabbage (Brassica rapa L.) parental line Nou 6 gou. Breed. Sci. 61: 151-159.

Kawamura, K., T. Kawanabe, M. Shimizu, A. J. Nagano, N. Saeki, K. Okazaki, M. Kaji, E. S. Dennis, K. Osabe and R. Fujimoto. 2016. Genetic distance of inbred lines of Chinese cabbage and its relationship to heterosis. Plant Gene 5: 1-7.

Kawanabe, T., K. Osabe, E. Itabashi, K. Okazaki, E. S. Dennis and R. Fujimoto. 2016. Development of primer sets that can verify the enrichment of histone modifications, and their application to examining vernalization-mediated chromatin changes in Brassica rapa L. Genes Genet. Syst. 91: 1-10.

Kim, D., G. Pertea, C. Trapnell, H. Pimentel, R. Kelley and S. L. Salzberg. 2013. TopHat2: accurate alignment of transcriptomes in the presence of insertions, deletions and gene fusions. Genome Biol. 14: R36. DOI: 10.1186/gb-2013-14-4r36.

Kim, D. H. and S. Sung. 2010. The Plant Homeo Domain finger protein, VIN3-LIKE 2, is necessary for photoperiodmediated epigenetic regulation of the floral repressor, MAF5. Proc. Natl. Acad. Sci. USA 107: 17029-17034.

Kim, D. H. and S. Sung. 2014. Genetic and epigenetic mechanisms underlying vernalization. The Arabidopsis Book 12: e0171. DOI: 10.1199/tab.0171.

Kim, S. Y., B. S. Park, S. J. Kwon, J. Kim, M. H. Lim, Y. D. Park, D. Y. Kim, S. C. Suh, Y. M. Jin, J. H. Ahn and Y. H. Lee. 2007. Delayed flowering time in Arabidopsis and Brassica rapa by the overexpression of FLOWERING LOCUS C (FLC) homologs isolated from Chinese cabbage (Brassica rapa L. ssp. pekinensis). Plant Cell Rep. 26: 327-336.

Kitamoto, N., S. Yui, K. Nishikawa, Y. Takahata and S. Yokoi. 2014. A naturally occurring long insertion in the first intron in the Brassica rapa FLC2 gene causes delayed bolting. Euphytica 196: 213-223.

Kole, C., P. Quijada, S. D. Michaels, R. M. Amasino and T. C. Osborn. 2001. Evidence for homology of flowering-time genes VFR2 from Brassica rapa and FLC from Arabidopsis thaliana. Theor. Appl. Genet. 102: 425-430.

Levy, Y. Y., S. Mesnage, J. S. Mylne, A. R. Gendall and C. Dean. 2002. Multiple roles of Arabidopsis VRN1 in vernalization and flowering time control. Science 297: 243-246.

Li, F., H. Kitashiba, K. Inaba and T. Nishio. 2009. A Brassica rapa linkage map of EST-based SNP markers for identification of candidate genes controlling flowering time and leaf morphological traits. DNA Res. 16: 311-323.

Li, X., S. Zhang, J. Bai and Y. He. 2016. Tuning growth cycles of Brassica crops via natural antisense transcripts of BrFLC. Plant Biotechnol. J. 14: 905-914.

Liu, C., S. Wang, W. Xu and X. Liu. 2017. Genome-wide transcriptome profiling of radish (Raphanus sativus L.) in response to vernalization. PLoS One 12: e0177594. DOI: 10.1371/journal.pone.0177594.

Long, W., X. Zou and X. Zhang. 2015. Transcriptome analysis of canola (Brassica napus) under salt stress at the germination stage. PLoS One 10: e0116217. DOI: 10.1371/journal.pone. 0116217.

Lou, P., J. Zhao, J. S. Kim, S. Shen, D. P. Del Carpio, X. Song, M. Jin, D. Vreugdenhil, X. Wang, M. Koornneef and G. Bonnema. 2007. Quantitative trait loci for flowering time and morphological traits in multiple populations of Brassica rapa. J. Exp. Bot. 58: 4005-4016.

Lyons, R., A. Rusu, J. Stiller, J. Powell, J. M. Manners and K. Kazan. 2015. Investigating the association between flowering time and defense in the Arabidopsis thaliana-Fusarium oxysporum interaction. PLoS One 10: e0127699. DOI: 10.1371/journal.pone.0127699.

Michaels, S. D. and R. M. Amasino. 1999. FLOWERING LOCUS $C$ encodes a novel MADS domain protein that acts as a repressor of flowering. Plant Cell 11: 949-956.

Michaels, S. D. and R. M. Amasino. 2001. Loss of FLOWERING LOCUS $C$ activity eliminates the late-flowering phenotype of FRIGIDA and autonomous pathway mutations but not responsiveness to vernalization. Plant Cell 13: 935-941.

Miyaji, N., M. Shimizu, J. Miyazaki, K. Osabe, M. Sato, Y. Ebe, S. Takada, M. Kaji, E. S. Dennis, R. Fujimoto and K. Okazaki. 2017. Comparison of transcriptome profiles by Fusarium oxysporum inoculation between Fusarium yellows resistant and susceptible lines in Brassica rapa L.. Plant Cell Rep. 36: 1841-1854.

Mortazavi, A., B. A. Williams, K. McCue, L. Schaeffer and B. Wold. 2008. Mapping and quantifying mammalian transcriptomes by RNA-Seq. Nat. Methods 5: 621-628.

Nagano, A. J., M. N. Honjo, M. Mihara, M. Sato and H. Kudoh. 2015. Detection of plant viruses in natural environments by using RNA-Seq. Methods Mol. Biol. 1236: 89-98.

Oh, S., H. Zhang, P. Ludwig and S. van Nocker. 2004. A mechanism related to the yeast transcriptional regulator Paflc is required for expression of the Arabidopsis FLC/MAF MADS box gene family. Plant Cell 16: 2940-2953.

Osborn, T. C., C. Kole, I. A. P. Parkin, A. G. Sharpe, M. Kuiper, D. J. Lydiate and M. Trick. 1997. Comparison of flowering time genes in Brassica rapa, B.napus and Arabidopsis thaliana. Genetics 146: 1123-1129.

Ratcliffe, O. J., R. W. Kumimoto, B. J. Wong and J. L. Riechmann. 2003. Analysis of the Arabidopsis MADS AFFECTING FLOWERING gene family: MAF2 prevents vernalization by short periods of cold. Plant Cell 15: 11591169.

Ratcliffe, O. J., G. C. Nadzan, T. L. Reuber and J. L. Riechmann. 2001. Regulation of flowering in Arabidopsis by an FLC homologue. Plant Physiol. 126: 122-132.

Saeki, N., T. Kawanabe, H. Ying, M. Shimizu, M. Kojima, H. Abe, K. Okazaki, M. Kaji, J. M. Taylor, H. Sakakibara, W. J. Peacock, E. S. Dennis and R. Fujimoto. 2016. Molecular and cellular characteristics of hybrid vigour in a commercial hybrid of Chinese cabbage. BMC Plant Biol. 16: 45. DOI: 10.1186/s12870-016-0734-3.

Schranz, M. E., P. Quijada, S. B. Sung, L. Lukens, R. Amasino and T. C. Osborn. 2002. Characterization and effects of the replicated flowering time gene FLC in Brassica rapa. Genetics 162: 1457-1468.

Scortecci, K. C., S. D. Michaels and R. M. Amasino. 2001. Identification of a MADS-box gene, FLOWERING LOCUS M, that represses flowering. Plant J. 26: 229-236.

Scortecci, K., S. D. Michaels and R. M. Amasino. 2003. Genetic 
interactions between FLM and other flowering-time genes in Arabidopsis thaliana. Plant Mol. Biol. 52: 915-922.

Shea, D. J., E. Itabashi, S. Takada, E. Fukai, T. Kakizaki, R. Fujimoto and K. Okazaki. 2018. The role of FLOWERING LOCUS C in vernalization of Brassica: the importance of vernalization research in the face of climate change. Crop Pasture Sci. 69: 30-39.

Shea, D. J., N. Nishida, S. Takada, E. Itabashi, S. Takahashi, A. Akter, N. Miyaji, K. Osabe, H. Mehraj, M. Shimizu, M. Seki, T. Kakizaki, K. Okazaki, E. S. Dennis and R. Fujimoto. 2019. Long noncoding RNAs in Brassica rapa L. following vernalization. Sci. Rep. 9: 9302. DOI: 10.1038/ s41598-019-45650-w.

Sheldon, C. C., J. E. Burn, P. P. Perez, J. Metzger, J. A. Edwards, W. J. Peacock and E. S. Dennis. 1999. The FLF MADS box gene: a repressor of flowering in Arabidopsis regulated by vernalization and methylation. Plant Cell 11: 445-458.

Sheldon, C. C., E. J. Finnegan, E. S. Dennis and W. J. Peacock. 2006. Quantitative effects of vernalization on $F L C$ and SOC1 expression. Plant J. 45: 871-883.

Sheldon, C. C., E. J. Finnegan, W. J. Peacock and E. S. Dennis. 2009. Mechanisms of gene repression by vernalization in Arabidopsis. Plant J. 59: 488-498.

Sheldon, C. C., D. T. Rouse, E. J. Finnegan, W. J. Peacock and E. S. Dennis. 2000. The molecular basis of vernalization: the central role of FLOWERING LOCUS C (FLC). Proc. Natl. Acad. Sci. USA 97: 3753-3758.

Shimizu, M., R. Fujimoto, H. Ying, Z. J. Pu, Y. Ebe, T. Kawanabe, N. Saeki, J. M. Taylor, M. Kaji, E. S. Dennis and K. Okazaki. 2014. Identification of candidate genes for fusarium yellows resistance in Chinese cabbage by differential expression analysis. Plant Mol. Biol. 85: 247-257.

Su, T., W. Wang, P. Li, B. Zhang, P. Li, X. Xin, H. Sun, Y. Yu, D. Zhang, X. Zhao, C. Wen, G. Zhou, Y. Wang, H. Zheng, S. $\mathrm{Yu}$ and F. Zhang. 2018. A genomic variation map provides insights into the genetic basis of spring Chinese cabbage (Brassica rapa ssp. pekinensis) selection. Mol. Plant 11: 1360-1376.

Sun, M., X. Qi, L. Hou, X. Xu, Z. Zhu and M. Li. 2015. Gene expression analysis of pak choi in response to vernalization. PLoS One 10: e0141446. DOI: 10.1371/journal.pone. 0141446.

Sung, S. and R. M. Amasino. 2004. Vernalization in Arabidopsis thaliana is mediated by the PHD finger protein VIN3. Nature 427: 159-164.

Swindell, W. R., M. Huebner and A. P. Weber. 2007. Transcriptional profiling of Arabidopsis heat shock proteins and transcription factors reveals extensive overlap between heat and non-heat stress response pathways. BMC Genomics 8: 125 . DOI: $10.1186 / 1471-2164-8-125$.

Takada, S., A. Akter, E. Itabashi, N. Nishida, D. J. Shea, N. Miyaji, H. Mehraj, K. Osabe, M. Shimizu, T. TakasakiYasuda, T. Kakizaki, K. Okazaki, E. S. Dennis and R. Fujimoto. 2019. The role of FRIGIDA and FLOWERING LOCUS C genes in flowering time of Brassica rapa leafy vegetables. Sci. Rep. 9: 13843. DOI: 10.101038/s41598019-50122-2.

Trapnell, C., A. Roberts, L. Goff, G. Pertea, D. Kim, D. R. Kelley, H. Pimentel, S. L. Salzberg, J. L. Rinn and L. Pachter. 2012. Differential gene and transcript expression analysis of RNA-seq experiments with TopHat and Cufflinks. Nat. Protoc. 7: 562-578.
Wang, A., J. Hu, X. Huang, X. Li, G. Zhou and Z. Yan. 2016. Comparative transcriptome analysis reveals heat-responsive genes in Chinese cabbage (Brassica rapa ssp. chinensis). Front. Plant Sci. 7: 939. DOI: 10.3389/fpls.2016.00939.

Wang, J., Y. Qiu, F. Cheng, X. Chen, X. Zhang, H. Wang, J. Song, M. Duan, H. Yang and X. Li. 2017. Genome-wide identification, characterization, and evolutionary analysis of flowering genes in radish (Raphanus sativus L.). BMC Genomics 18: 981. DOI: 10.1186/s12864-017-4377-z.

Wang, X., H. Wang, J. Wang, R. Sun, J. Wu, S. Liu, Y. Bai, J. H. Mun, I. Bancroft, F. Cheng, S. Huang, X. Li, W. Hua, J. Wang, X. Wang, M. Freeling, J. C. Pires, A. H. Paterson, B. Chalhoub, B. Wang, A. Hayward, A. G. Sharpe, B. S. Park, B. Weisshaar, B. Liu, B. Li, B. Liu, C. Tong, C. Song, C. Duran, C. Peng, C. Geng, C. Koh, C. Lin, D. Edwards, D. $\mathrm{Mu}, \mathrm{D}$. Shen, E. Soumpourou, F. Li, F. Fraser, G. Conant, G. Lassalle, G. J. King, G. Bonnema, H. Tang, H. Wang, H. Belcram, H. Zhou, H. Hirakawa, H. Abe, H. Guo, H. Wang, H. Jin, I. A. Parkin, J. Batley, J. S. Kim, J. Just, J. Li, J. Xu, J. Deng, J. A. Kim, J. Li, J. Yu, J. Meng, J. Wang, J. Min, J. Poulain, J. Wang, K. Hatakeyama, K. Wu, L. Wang, L. Fang, M. Trick, M. G. Links, M. Zhao, M. Jin, N. Ramchiary, N. Drou, P. J. Berkman, Q. Cai, Q. Huang, R. Li, S. Tabata, S. Cheng, S. Zhang, S. Zhang, S. Huang, S. Sato, S. Sun, S. J. Kwon, S. R. Choi, T. H. Lee, W. Fan, X. Zhao, X. Tan, X. Xu, Y. Wang, Y. Qiu, Y. Yin, Y. Li, Y. Du, Y. Liao, Y. Lim, Y. Narusaka, Y. Wang, Z. Wang, Z. Li, Z. Wang, Z. Xiong and Z. Zhang. 2011. The genome of the mesopolyploid crop species Brassica rapa. Nat. Genet. 43: 1035-1039.

Wood, C. C., M. Robertson, G. Tanner, W. J. Peacock, E. S. Dennis and C. A. Helliwell. 2006. The Arabidopsis thaliana vernalization response requires a polycomb-like protein complex that also includes VERNALIZATION INSENSITIVE 3. Proc. Natl. Acad. Sci. USA 103: 14631-14636.

Xiao, D., J. J. Zhao, X. L. Hou, R. K. Basnet, D. P D. Carpio, N. W. Zhang, J. Bucher, K. Lin, F. Cheng, X. W. Wang and G. Bonnema. 2013. The Brassica rapa FLC homologue $F L C 2$ is a key regulator of flowering time, identified through transcriptional co-expression networks. J. Exp. Bot. 64: 4503-4516.

Xing, M., H. Lv, J. Ma, D. Xu, H. Li, L. Yang, J. Kang, X. Wang and Z. Fang. 2016. Transcriptome profiling of resistance to Fusarium oxysporum f. sp. conglutinans in cabbage (Brassica oleracea) roots. PLoS One 11: e0148048. DOI: 10.1371/journal.pone.0148048.

Yong, H. Y., Z. Zou, E. P. Kok, B. H. Kwan, K. Chow, S. Nasu, M. Nanzyo, H. Kitashiba and T. Nishio. 2014. Comparative transcriptome analysis of leaves and roots in response to sudden increase in salinity in Brassica napus by RNA-seq. BioMed Res. Int. 2014: 1-19.

Zhang, H. and S. van Nocker. 2002. The VERNALIZATION INDEPENDENCE 4 gene encodes a novel regulator of FLOWERING LOCUS C. Plant J. 31: 663-667.

Zhao, J., V. Kulkarni, N. Liu, D. P. Del Carpio, J. Bucher and G. Bonnema. 2010. BrFLC2 (FLOWERING LOCUS C) as a candidate gene for a vernalization response QTL in Brassica rapa. J. Exp. Bot. 61: 1817-1825.

Zou, X., X. Tan, C. Hu, L. Zeng, G. Lu, G. Fu, Y. Cheng and X. Zhang. 2013. The transcriptome of Brassica napus L. roots under waterlogging at the seedling stage. Int. J. Mol. Sci. 14: 2637-2651. 